\title{
On the formation of non-topological string networks
}

\author{
Ana Achúcarro \\ Department of Theoretical Physics, UPV-EHU, Bilbao, Spain \\ Institute for Theoretical Physics, University of Groningen, The Netherlands \\ Julian Borrill \\ Center for Particle Astrophysics, University of California, Berkeley, CA 94720 \\ National Energy Research Scientific Computing Center, Lawrence Berkeley National Laboratory, University of California, \\ Berkeley, CA 94720 \\ Andrew R. Liddle \\ Astronomy Centre, University of Sussex, Falmer, Brighton BN1 9QJ, United Kingdom
}

(October 17, 2018)

\begin{abstract}
We review recent simulations of the formation of a particular class of non-topological defects known as semilocal strings during a phase transition. Semilocal strings have properties that are intermediate between topological cosmic strings and stable electroweak strings, and therefore the observation that they could form in substantial quantities during a cosmological phase transition has implications for structure formation, baryogenesis, etc. At the same time, and from a purely theoretical point of view, they provide a very good testing ground for investigating the role of gauge fields in defect formation.
\end{abstract}

PACS numbers: 11.27.+d, 11.15.Ex

\section{INTRODUCTION}

Our current understanding of particle physics and cosmology implies that the early Universe probably went through a series of symmetry breaking phase transitions as it cooled down and expanded to become what we know today. In these transitions topological (and possibly non-topological) defects are expected to have formed [1,2]. Although the cosmological evidence for the existence of such defects remains unclear [3], there is plenty of experimental evidence from condensed matter systems that networks of defects do form in symmetry breaking phase transitions according to the Kibble-Zurek mechanism [1, [, 5], the most recent confirmation coming from the experiments in vortex formation in superfluid Helium that have been performed here in Helsinki at the ULTI and in Grenoble and Lancaster [6.

From the theoretical point of view, the last few years have seen great progress in our understanding of the creation of topological defects in a symmetry breaking phase transition, largely through the interaction between cosmologists and condensed matter physicists in a beautiful example of interdisciplinary collaboration [5]. However, it is fair to say that so far most of this progress has been in cases involving only scalar fields and global symmetries, whereas most theories of elementary particles are based on gauge, rather than global, symmetries, and it is these models that are relevant to cosmology.

Defect formation in these systems remains an open problem. For a start, the usual order parameter (the expectation value of the Higgs) is not gauge-invariant, and it is not obvious in general how to choose a gaugeinvariant order parameter. The applicability of the geodesic rule is also under scrutiny [7,8]. At a more basic level, even the nature of the electroweak phase transition is under discussion. Under the circumstances, it is difficult to make reliable estimates of the initial density of gauged defects such as electroweak [9] or semilocal [10,11] strings, which is of course a necessary first step towards understanding their cosmological implications for structure formation, baryogenesis or generation of primordial magnetic fields.

Gauge fields are often neglected in analytic calculations, largely because the usual techniques do not lend themselves easily to their inclusion. But if we are to believe numerical simulations we have to conclude that this may not be such a good idea; whenever present, gauge fields tend to play a crucial role in the dynamics. A rather drastic example is the existence of nonhomogeneous ground states (even in the absence of external magnetic fields) in large samples of certain twodimensional unconventional superconductors whose order parameter has a magnetic moment. An incommensurate phase appears which can be described as a parallel array of (non-topological) domain walls; the magnetic field lives in the core of these walls, where there is symmetry restoration, with the magnetic flux alternating in sign from one wall to the next so that the net magnetic flux through the sample remains zero. These inhomogeneous phases have not been observed experimentally, as there is no known system in the right range of values of the Landau-Ginzburg parameters, but the theoretical 
prediction is well established [12].

Another example of the effect of gauge fields on the dynamics is the observed breakdown of the geodesic rule (the assumption that the field configuration will take the shortest interpolating path between different vacuum states) during first order phase transitions in systems with gauge invariance [7, 8]. A recent numerical study by Copeland and Saffin, both in the case of the abelian Higgs model and the $\theta_{w}=0$ limit of the Weinberg-Salam model, has found there may be enough energy in the collisions of separated nucleated bubbles to drive the Higgs value away from its vacuum expectation value and make it oscillate around zero, so extra regions with symmetry restoration are produced. The validity of the geodesic rule seems to depend not only on the initial bubble separation but also on the gauge coupling, indicating gauge fields are playing a crucial role.

The upshot of both studies would seem to be that the backreaction of the gauge fields on the scalars can result in an enhancement of symmetry restoration leading to larger defect densities than one would have inferred from the scalar fields alone. Here we present recent results 13, 14 that corroborate this idea in the context of electroweak strings.

In particular, we have studied the formation of a type of non-topological vortices known as semilocal strings; these are stable Abrikosov-Nielsen-Olesen vortices in the $\theta_{w} \rightarrow \pi / 2$ limit of the (bosonic sector of the) WeinbergSalam model [10]. Note that in these models the vacuum manifold -the manifold of ground states- is simply connected thus if one ignored the effect of gauge fields one would not expect any vortices to be formed at all.

Before we continue, we should point out that analogous systems have been studied in condensed matter. In [15, the system was an unconventional superconductor where the global $\mathrm{SU}(2)$ group was the spin rotation group. In [16] the hypothetical case of an "electrically charged" Aphase of $3 \mathrm{He}$ (a superconductor with the properties of $3 \mathrm{He}-\mathrm{A})$ was considered. In this case the global group was $\mathrm{SO}(3)$, the group of orbital rotations. Both papers discussed textures (continuous vortices) in such superconductors, which correspond to the "skyrmionic" configurations of 11.25] (see Fig. 1).

We have carried out a numerical study of semilocal string formation through the Kibble-Zurek mechanism based on a novel numerical technique 13, 14] which can be applied to other gauged non-topological defects as well. The strategy we propose is to follow the evolution of the field strength, which is used as an indicator for the presence of defects; the initial conditions are obtained by an extension of the Vachaspati-Vilenkin algorithm [17] appropriate to non-topological defects, plus a short period of dynamical evolution including a dissipation term (numerical viscosity) to aid the relaxation of configurations in the 'basin of attraction' of the defects under study.

We observe that, even if we assume no symmetry restoration in the initial stages of the phase transition, the interaction between the gauge fields and the scalar fields is such that symmetry restoration will eventually occur simply because it is energetically favourable for the magnetic field to be concentrated in regions where the Higgs field has a value close to that of the symmetric phase. Thus, vortices are formed.

Since we are proposing a new technique, we test it in several ways. Restriction to the abelian Higgs model gives excellent agreement with analytic and numerical estimates for cosmic strings, a simpler type of topological defect analogous to a superconduting flux-tube, as given in 17], and we also test the robustness of our results under varying initial conditions and numerical viscosity. Finally, our results seem to be in good agreement with previous estimates for semilocal string formation in 18, 19.

It is worth mentioning that our analysis suggests a similar behaviour for electroweak strings away from $\theta_{w}=\pi / 2$, as long as they are stable. Nagasawa and Yokoyama 20] estimated the initial density of (unstable) electroweak strings by looking at the thermal distribution of scalar fields and concluded that the density would be negligible. While their calculation at $\sin ^{2} \theta_{w} \sim 0.23$ is not necessarily in contradiction with ours at $\theta_{w}=\pi / 2$, their method neglects the role of the gauge fields, which we would argue to be a key ingredient. Thus, full numerical simulation appears to us to be the only reliable way to investigate electroweak string formation rates.

In what follows we briefly summarize our technique and results. For more information we refer the reader to our papers [13, 14, 21 and web pages. Colour images and movies of the three-dimensional simulations can currently be found on the WWW at http://cfpa.berkeley.edu/ borrill/defects/semilocal.htm. Also, a 100 frame mpeg movie $(0.5 \mathrm{Mb})$ of the twodimensional simulation can be viewed at http://star-www.cpes.susx.ac.uk/people/arl_recent.htm.

\section{THE MODEL}

We work in flat space-time throughout. The semilocal model is described by the following lagrangian:

$$
\begin{aligned}
\mathcal{L}= & \left(\nabla_{\mu}-i e A_{\mu}\right) \phi_{1}^{\dagger}\left(\nabla^{\mu}+i e A^{\mu}\right) \phi_{1} \\
& +\left(\nabla_{\mu}-i e A_{\mu}\right) \phi_{2}^{\dagger}\left(\nabla^{\mu}+i e A^{\mu}\right) \phi_{2} \\
& -\frac{1}{4} F_{\mu \nu} F^{\mu \nu}-\frac{\lambda}{2}\left(\left|\phi_{1}\right|^{2}+\left|\phi_{2}\right|^{2}-\frac{\eta^{2}}{2}\right)^{2},
\end{aligned}
$$

where $\phi_{1}$ and $\phi_{2}$ are two equally-charged complex scalar fields, $A_{\mu}$ is a U(1) gauge field and $F_{\mu \nu}=\nabla_{\mu} A_{\nu}-\nabla_{\nu} A_{\mu}$ the associated gauge field strength. Notice that this is just the bosonic sector of the Weinberg-Salam model in the $\theta_{w}=\pi / 2$ limit in which the $\mathrm{SU}(2)$ coupling constant 
is set to zero and the $\mathrm{W}$-bosons decouple. In particular its vacuum manifold is the three-sphere $S^{3}$, which has no non-contractible loops, and yet stable AbrikosovNielsen-Olesen vortices [22,23] can form 10,11]. On the other hand, setting $\phi_{2}=0$ obtains the abelian Higgs model; thus, comparison with topological strings is straightforward, and we will use it repeatedly as a test case, both to check our simulation techniques and to minimize systematic errors when quoting formation rates.

As in the abelian Higgs model, the gauge coupling and the vacuum expectation value of the Higgs can be rescaled to one by choosing appropriate units (the inverse vector mass, for length, and the symmetry breaking scale, for energy). In that case the Lagrangian becomes

$$
\begin{aligned}
\mathcal{L}= & \left(\nabla_{\mu}-i A_{\mu}\right) \phi_{1}^{\dagger}\left(\nabla^{\mu}+i A^{\mu}\right) \phi_{1} \\
& +\left(\nabla_{\mu}-i A_{\mu}\right) \phi_{2}^{\dagger}\left(\nabla^{\mu}+i A^{\mu}\right) \phi_{2} \\
& -\frac{1}{4} F_{\mu \nu} F^{\mu \nu}-\frac{\beta}{2}\left(\left|\phi_{1}\right|^{2}+\left|\phi_{2}\right|^{2}-1\right)^{2},
\end{aligned}
$$

and the only remaining parameter in the theory is $\beta=$ $m_{\mathrm{s}}^{2} / m_{\mathrm{v}}^{2}$, the ratio between the scalar and vector masses (squared), whose value determines the stability of an infinitely long, straight, string with a Nielsen-Olesen profile: it is stable for $\beta<1$, neutrally stable for $\beta=1$ and unstable for $\beta>1$ [10,11, 18]. For $\beta=1$ there is a family of solutions with the same energy and different core widths, of which only the semilocal string has complete symmetry restoration in the center [11,24].

Whenever $\beta<1$ we expect a certain amount of these non-topological strings to form during a phase transition; the lower the value of $\beta$, the higher the formation rate. The enhancement of symmetry restoration is easily understood from the minimal coupling between the gauge field and the scalars. In regions where the magnetic field is strong (because of fluctuations), the term $A^{2} \phi^{2}$ in the lagrangian will tend to drive the Higgs' expectation value towards zero, in competition with the potential term. This is basically the same physics as in the Meissner effect, the expulsion of magnetic fields from a superconductor. In finite samples subjected to an external magnetic field the system reacts by forming surface currents that screen the magnetic field, but if the magnetic field is strong enough it will penetrate the sample through regions where the symmetry is restored (e.g. Abrikosov vortices, in type II superconductors [22]). Similarly, in a cosmological context, the best way to cope with a strong fluctuation of the magnetic field is to reduce the value of $\Phi$ towards zero.

\section{NUMERICAL SIMULATIONS}

We work in temporal gauge $A_{0}=0$. Splitting up the scalar fields into four real scalars via $\phi_{1}=\psi_{1}+i \psi_{2}$, $\phi_{2}=\psi_{3}+i \psi_{4}$, the equations of motion are

$$
\begin{gathered}
\ddot{\psi}_{a}-\nabla^{2} \psi_{a}+\beta\left(\psi_{1}^{2}+\psi_{2}^{2}+\psi_{3}^{2}+\psi_{4}^{2}-1\right) \psi_{a} \\
+A^{2} \psi_{a}+(-1)^{b}(2 A \cdot \nabla+\nabla \cdot A) \psi_{b}=0,
\end{gathered}
$$

(where $b$ is the complement of $a-1 \leftrightarrow 2,3 \leftrightarrow 4$ and dots are time derivatives) for the scalar fields and

$$
\begin{array}{r}
\ddot{A}_{i}-\nabla^{2} A_{i}+\nabla_{i} \nabla \cdot A+2\left(\psi_{1} \overleftrightarrow{\nabla}_{i} \psi_{2}+\psi_{3} \overleftrightarrow{\nabla}_{i} \psi_{4}\right) \\
+2 A_{i}\left(\psi_{1}^{2}+\psi_{2}^{2}+\psi_{3}^{2}+\psi_{4}^{2}\right)=0,
\end{array}
$$

for the gauge fields $(i=1,2,3)$, together with Gauss' law, which here is a constraint derived from the gauge choice, and is used to test the stability of the code,

$$
2\left(\psi_{1} \overleftrightarrow{\nabla}_{0} \psi_{2}+\psi_{3} \overleftrightarrow{\nabla}_{0} \psi_{4}\right)+\nabla_{i} \dot{A}_{i}=0
$$

The arrows indicate asymmetric derivatives.

This system is discretized using a standard nuerical technique known as the staggered leapfrog method. However, to reduce its relaxation time we also add an $a d h o c$ dissipation term to each equation $\left(\eta \dot{\psi}_{a}\right.$ and $\eta \dot{A}_{i}$ respectively). In an expanding Universe the expansion rate would play such a role, though $\eta$ would typically not be constant. We tested a range of strengths of dissipation, and checked that it did not significantly affect the number densities obtained. The simulations we display later used $\eta=0.5$ and periodic boundary conditions.

We estimate the number density of defects by an extension of the Vachaspati-Vilenkin algorithm [17]: we first generate a random initial configuration for the scalar fields drawn from the vacuum manifold, which is not discretized, then find the gauge field configuration that minimizes the energy associated with (covariant) gradients. If space is a grid of dimension $N \times N \times N$, the correlation length is chosen to be some number $p$ of grid points ( $p=16$ in our simulations). To obtain a reasonably smooth configuration for the scalar fields, we throw down random vacuum values on a $\frac{N}{p} \times \frac{N}{p} \times \frac{N}{p}$ subgrid; the scalar field is then interpolated onto the full grid by bisection. Strings are always identified with the location of magnetic flux tubes. For cosmic strings this accurately reproduces the standard results 17.

For semilocal strings, on the other hand, the initial configurations thus generated have a complicated flux structure with extrema of different values (top panel of Fig. 1), and it is far from clear which of these, if any, might evolve to form Nielsen-Olesen vortices; we resolve

\footnotetext{
*In fact, the energy-minimization condition is redundant, since the early stages of dynamical evolution carry out this role anyway. Having checked that our results were independent of the initial conditions for the gauge fields, we used $A_{i}(\mathbf{x})=\psi_{1} \nabla_{i} \psi_{2}-\psi_{2} \nabla_{i} \psi_{1}+\psi_{3} \nabla_{i} \psi_{4}-\psi_{4} \nabla_{i} \psi_{3}$ as the initial gauge field configuration in our simulations.
} 
this ambiguity by numerically evolving the configurations forward in time. As anticipated, in the unstable regime $\beta>1$ the flux quickly dissipates leaving no strings. By contrast, in the stable regime $\beta<1$ stringlike features emerge when configurations in the "basin of attraction" of the semilocal string relax unambiguously into vortices (bottom panel of Fig. 1).

The correlation length in the simulations is only constrained to be larger than the size of the vortex cores, to avoid overlaps. This results in a minimal value of the parameter $\beta$ of around 0.05 \% . For each of seven different values of $\beta$, we take several initial configurations on a $64^{3}$ (or $128^{2}$ ) grid smoothed over every 16 grid-points. As expected, for $\beta<1$ we find a formation rate which depends on $\beta$, tending to zero as $\beta$ tends to 1 . The formation rate is lower than in the cosmic string case, but significant: at the smallest $\beta$ we were able to simulate, $\beta=0.05$, the rate is a fraction of order $1 / 3$ of that of cosmic strings. Our statistical results are derived from a large suite of simulations (700 in all) carried out on a $64^{3}$ grid using a SUN Ultra II workstation. We have also performed a large $256^{3}$ simulation on the Cray T3E at NERSC from which we have extracted the image in Fig. 4.

\section{RESULTS}

Before discussing the results in any more detail, a few comments are in order about the general procedure we are following. We checked our strategy on a twodimensional toy model obtained by ignoring one of the spatial coordinates [13].

We first tested the performance of our codes for the case of cosmic strings by ignoring one of the (complex) scalar fields, setting $\psi_{3}=\psi_{4}=0$. This makes the defects topological, and the flux tubes formed now map out the locations of winding in the scalar field configurations. We obtained a cosmic string number density

$$
n_{\mathrm{cs}}=0.32 \pm 0.02
$$

per correlation area, in perfect agreement with the analytic value of $1 / 3$ obtained with the standard VachaspatiVilenkin algorithm on a 2-dimensional square lattice without discretizing the vacuum manifold. The good agreement arises because the simulations are themselves carried out on a square lattice, and confirms that our identification of cosmic strings via the flux tube structure works extremely well

\footnotetext{
${ }^{\dagger}$ If $\beta$ is lowered further, the scalar string cores become too wide to fit into a correlation volume, in contradiction with the vacuum values assumed in a Vachaspati-Vilenkin algorithm.

¥ The error quoted is the standard error on the mean, but
}

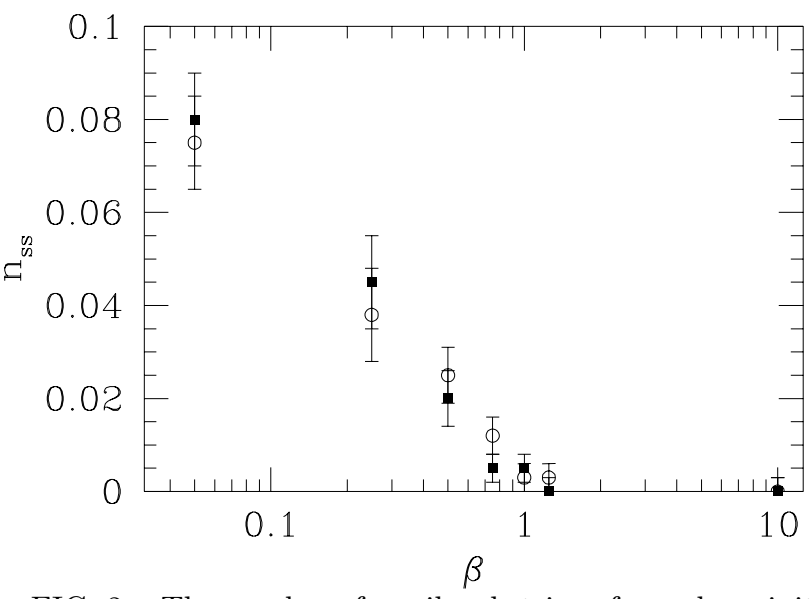

FIG. 2. The number of semilocal strings formed per initial two-dimensional correlation volume. Each point is an average over ten simulations. Squares indicate that the vacuum initial conditions described in the text were used, while open circles indicate that non-vacuum (thermal) initial conditions were used.

We also tested the robustness of our conclusions under varying initial conditions. We have already mentioned that the initial configuration for the gauge fields was seen to play no appreciable role. On the other hand, in a second-order phase transition one expects the scalar field to be out of the vacuum in some sort of thermal distribution. This has been studied by Ye and Brandenberger [27], and we closely followed their strategy, drawing the scalar field magnitude randomly from a gaussian distribution instead of the uniform distribution they use. Fig. 2 shows the results of two-dimensional simulations with two sets of initial conditions, one with no symmetry restoration and other with a more thermal distribution of scalar field values. We examined different choices for the width of the gaussian, and concluded that any reasonable choice makes no difference to the results; in those displayed in Fig. 2, the gaussian has a dispersion equal to the vacuum expectation value of the scalar field.

As can be seen, the influence of initial conditions is minimal. The same general shape for the $\beta$-dependence is found, and the number of strings identified per simulation is extremely close, with the error bars overlapping.

Our results are also in good agreement with those of [18], in which simple configurations some way away from the semilocal string were permitted to relax into semilocal strings. Finally, we note also that extrapolation of Fig. 2 to $\beta=0$ is consistent with the estimated value of

note that the uncertainty from discretization is somewhat larger: on a triangular lattice the Vachaspati-Vilenkin algorithm would give a formation rate of $1 / 4$ per triangle rather than $1 / 3$ per square 26], though one would also have to account for the difference in area. 

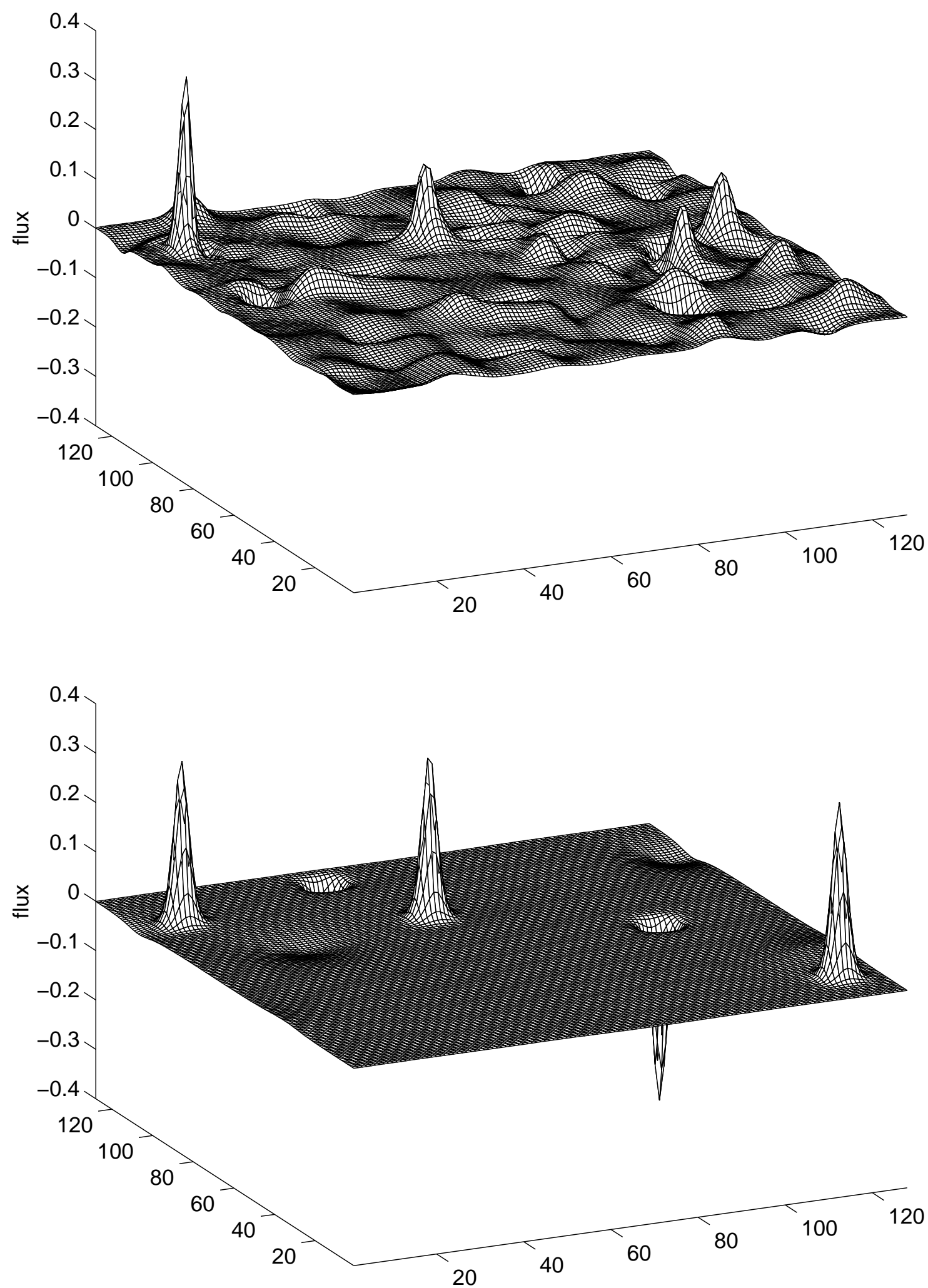

FIG. 1. The flux tube structure in a two-dimensional semilocal string simulation with $\beta=0.05$. The upper panel $(t=0)$ shows the initial condition after the process described in the text. The lower panel shows the configuration resolved into five flux tubes by a short period of dynamical evolution $(t=100)$. These flux tubes are semilocal vortices. Note the different numbers of upward and downward pointing flux tubes, despite the zero net flux boundary condition. The missing flux resides in the smaller 'nodules', made long-lived by the numerical viscosity; the expansion of the universe could have a similar effect and preserve these 'skyrmionic' configurations 25 . 
$1 / 8 \approx 0.12$ found in Ref. 19 .

This is a two-dimensional simulation, but it is also expected to be representative of any cross section of the three-dimensional one.

We can now proceed to describe the results of our simulation in some detail.

In order to bring down the statistical errors on our result we carried out a large number of runs; the counting of flux tubes was automated by adopting the criterion that any isolated extremum in the magnetic flux which was at least half that of a perfect Nielsen-Olesen vortex was identified as a string. We tested this criterion on selected simulations which we studied in detail ourselves, and concluded it gave an accurate counting.

As in the two-dimensional simulations (Fig. 1), the starting configurations obtained by our described procedure initially yield a complicated mess of flux. However, after a few timesteps the flux resolves itself into loops and open segments of string. We observed a clear interaction between nearby segments which join to form longer segments, as conjectured in [24,19]. Fig. 3 shows an open segment of string whose ends meet to form a loop.

Fig. 4 shows two time slices from a single large $\beta=0.05$ simulation using the Cray T3E at NERSC; these are zooms showing only part of the $256^{3}$ simulation box. We see a collection of short string segments and loops; visually this is very different from a cosmic string simulation where strings cannot have ends. As time progresses, the short segments either disappear or link up to form longer ones.

We can immediately conclude from these images that the formation rate of semilocal strings is not extremely close to zero; the fact that flux tubes are observed in our simulations implies that the formation rate cannot be much smaller than one per correlation volume.

In order to quantify the formation rate, we compute the total length of string in the simulations, always comparing the semilocal string density to that of a cosmic string simulation with the same properties (including dissipation) to minimize systematic errors. We determine the length by setting a magnetic flux threshold and computing the fractional volume of the box which exceeds it. In Fig. 5, we plot the length of semilocal string relative to the length found in cosmic string simulations, as a function of time and with $\beta=0.05$. We see that after a transient during which the initial tangle of flux sorts itself out, the system settles down to a reasonable equilibrium. The upward trend appears to be caused by the periodicity of the simulation box, which freezes-in any string crossing the box, favouring cosmic string annihilation because of their higher density. We take the relative densities of semilocal and cosmic strings to be that at time 50 in these simulations. There is a modest dependence on the choice of flux threshold, and we set it at one-half the flux density of a Nielsen-Olesen vortex.

Fig. 6 shows the resulting ratio of semilocal and cos-
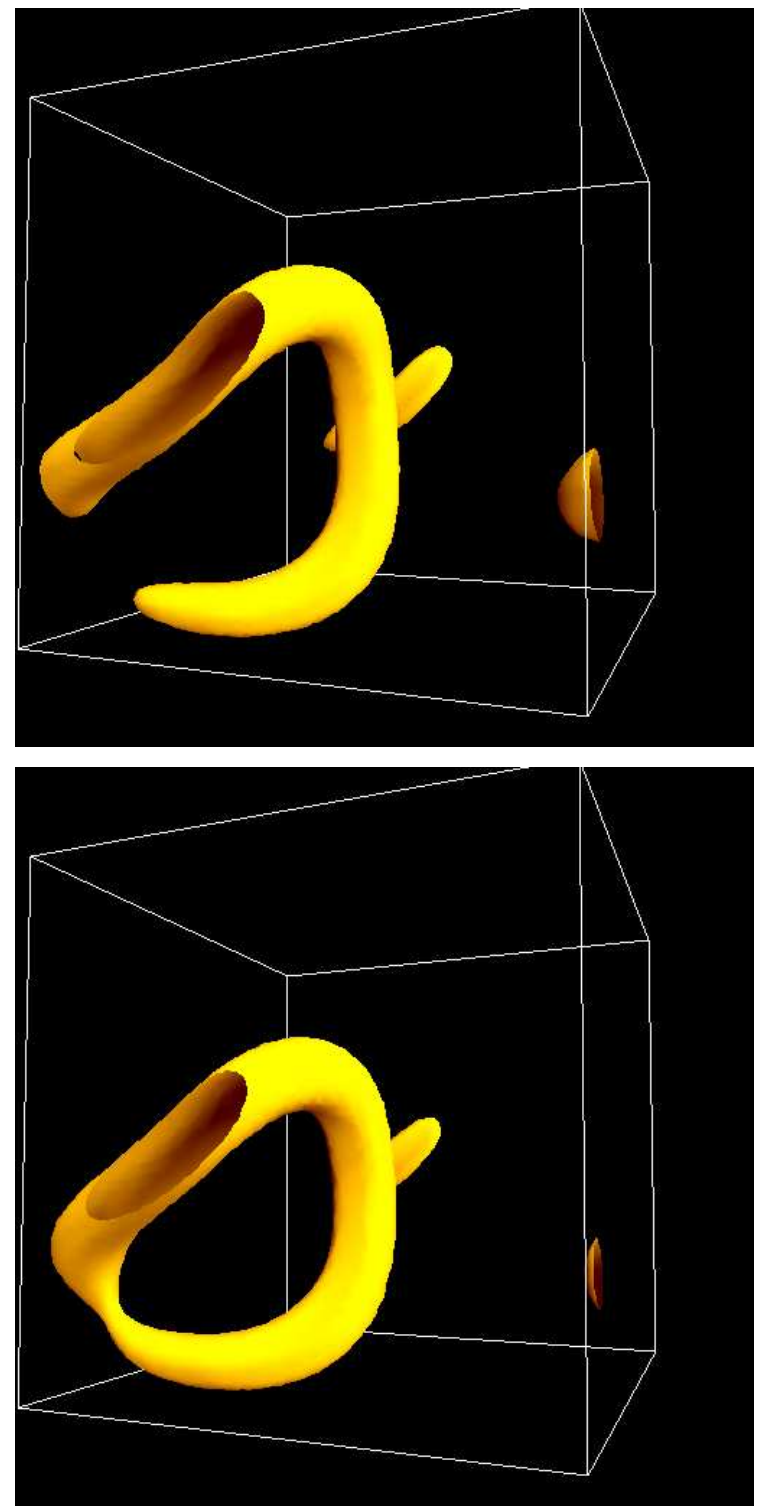

FIG. 3. Two snapshots, at $t=70$ and $t=80$, of a $64^{3}$ simulation with $\beta=0.05$ where the ends of an open segment of string join up to form a closed loop. The loops in our simulation seem to behave like those of topological cosmic string, contracting and disappearing.

mic string lengths, as a function of the stability parameter $\beta$. These results are derived from 50 simulations (both semilocal and cosmic) at each $\beta$ value, carried out in boxes of dimension $64^{3}$. The error bars include the statistical spread between simulations, and an estimated $25 \%$ systematic error from the length counting algorithm (see the spread in Fig. 5) and the viscosity. Those latter uncertainties are the dominant ones. Recalling that the formation rate of cosmic strings is estimated to be of order one per correlation volume (0.88 in Ref. [17]), these results are in excellent agreement with the twodimensional results we found in Fig. 2. They show a significant formation rate for low $\beta$, decreasing dramat- 


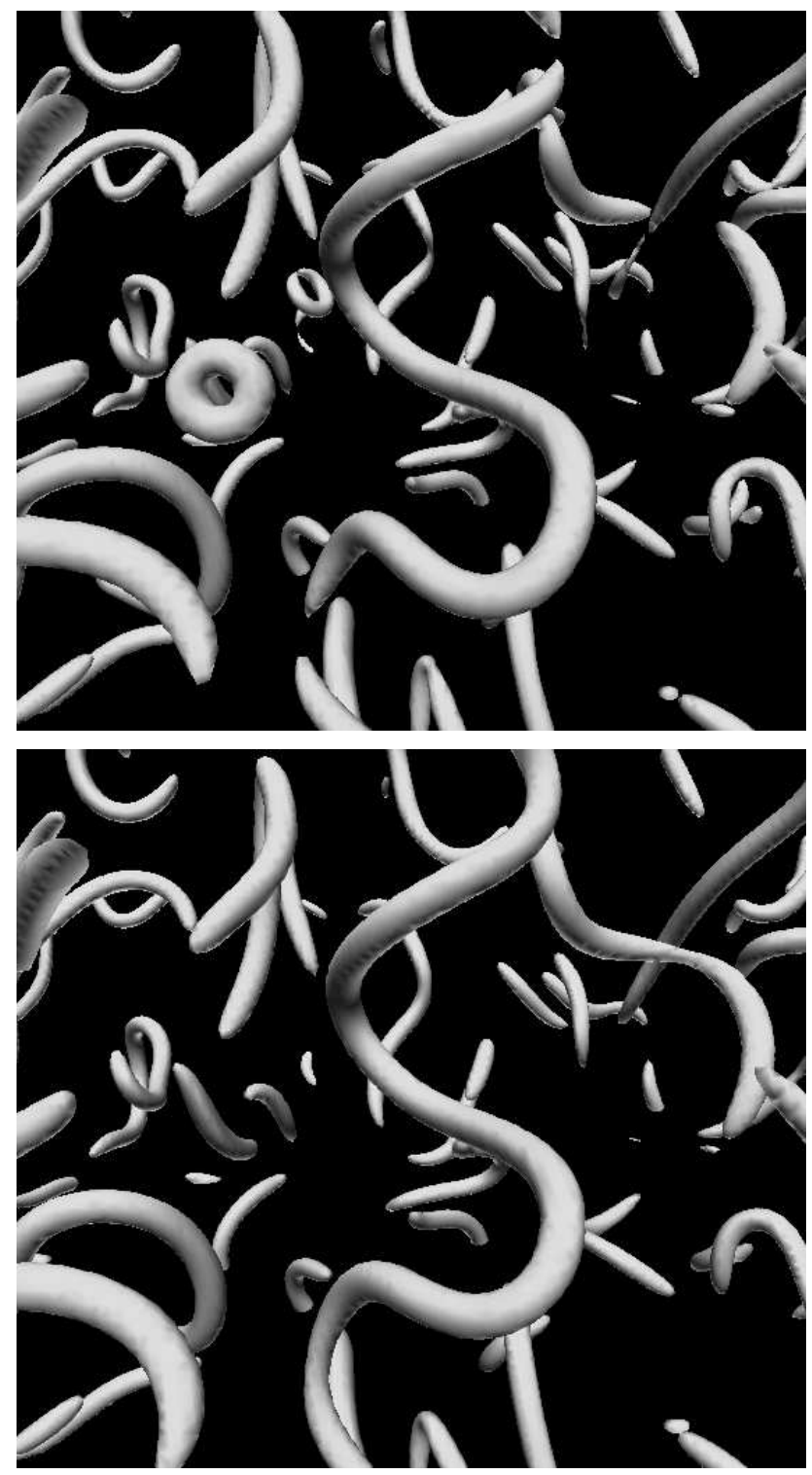

FIG. 4. Part of the large simulation, shown at time 60 and time 70. Note several joinings of string segments, e.g. two separate joinings on the long central string, and the disappearance of some loops. The different apparent thickness of strings is entirely an effect of perspective.

ically as $\beta \rightarrow 1$, beyond which there is no evidence of semilocal string formation. The small amount of string seen in some large- $\beta$ simulations is an artifact of the viscosity; the flux all dissipates if the viscosity is turned off, while it persists if $\beta<1$.

\section{CONCLUSIONS}

The formation of defects during a symmetry-breaking phase transition in systems with gauge invariance is a very important issue in cosmology, but it is not as well understood as it is in systems with only global symme-

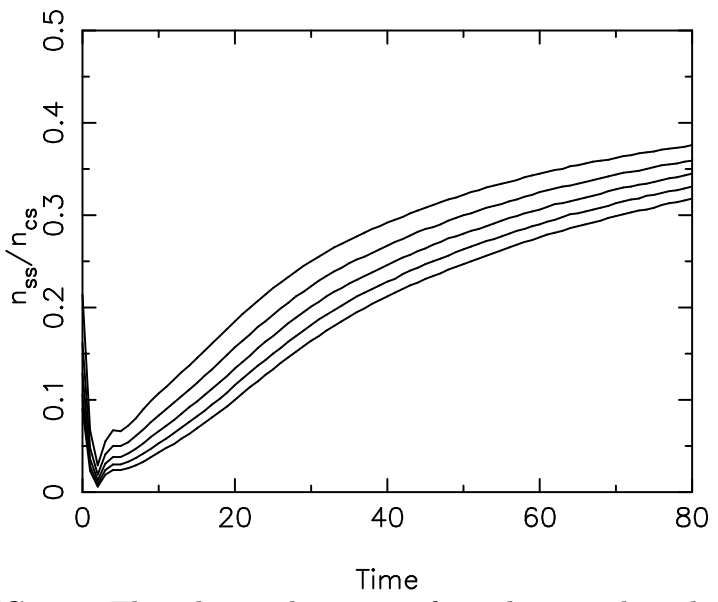

FIG. 5. This shows the ratio of total string lengths in a semilocal and cosmic string simulation, with $\beta=0.05$. The different lines show different magnetic flux thresholds, from bottom to top they are $0.6,0.55,0.5,0.45$ and 0.4 times the peak flux of a Nielsen-Olesen vortex.

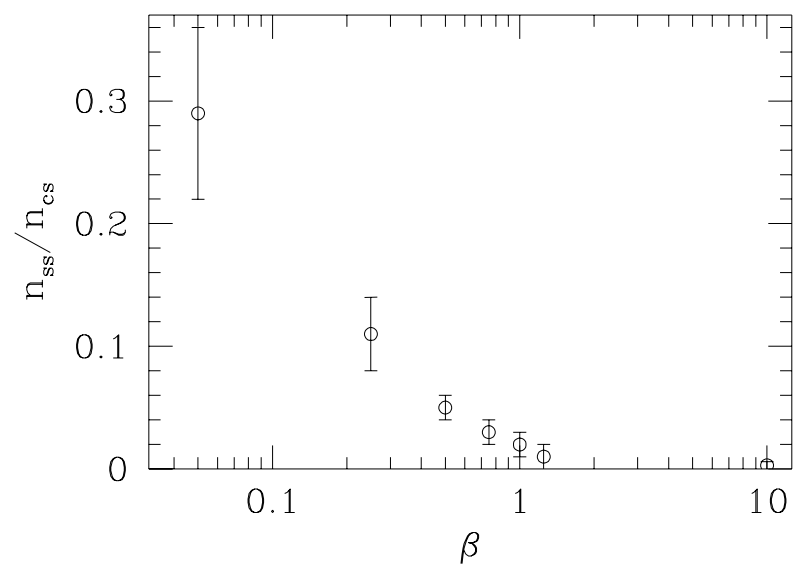

FIG. 6. The ratio of lengths of semilocal and cosmic strings.

tries. The lack of experimental data from condensed matter systems forces us to rely on numerical simulations to test our ideas. We have presented here a study of nontopological defect formation where the role of gauge fields is seen to be crucial and leads to a very different scenario from what one would have inferred from the scalar fields alone.

Our simulations have shown that dynamically stable non-topological defects, such as semilocal strings, could form in substantial quantities during a cosmological phase transition. This seems to happen even if the configuration immediately after the phase transition has no appreciable symmetry restoration (recall our initial conditions place the scalar field in the vacuum everywhere), and is due to the back-reaction of the gauge fields on the scalars, which favours a low vacuum expectation value for the latter. In the case of non-topological defects, the intensity of the effect, and therefore the number density 
of defects created, increases with the classical stability of the configurations. For semilocal strings with $\beta=0.05$ we found a formation rate as high as one third of that of topological strings, while as $\beta$ was increased towards the stability/instability transition at $\beta=1$, the density dropped to zero.

Moreover we have observed short segments of string growing and joining to form longer strings and loops, as conjectured in 19,24, while others collapsed longitudinally, as invoked in some baryogenesis scenarios [28]. The details of these dynamics seem to depend on the length of the segments and the distance between neighbouring ones and deserve further study, as do the scaling behaviour of the network and the implications for structure formation, baryogenesis, etc.

We suspect that a similar behaviour is to be expected of (stable) electroweak strings. Nagasawa and Yokoyama [20] studied electroweak string formation and concluded that the initial density would be totally negligible for realistic values of the weak mixing angle. However, this is not necessarily in contradiction with our results for two reasons. First of all, in our simulation semilocal strings arise during the evolution because of the back-reaction of the gauge fields on the scalars, which enables initially short pieces of string to grow and join up to form longer ones, an effect not included in their analysis. Secondly, their calculation takes place at $\sin ^{2} \theta_{w} \sim 0.23$, for which the electroweak string is dynamically unstable [29]; in this case we would find that all the flux dissipates soon after the phase transition. In any case, numerical simulation appears to be the only reliable way to address this problem.

In the meantime, the semilocal string model remains an excellent testing ground for defect formation scenarios in the presence of gauge symmetries.

\section{ACKNOWLEDGMENTS}

A.A. would like to thank the organizers of the conference, and in particular A. Babkin, M. Krusius and G. Volovik for the invitation to give this talk. J.B. was supported by the Laboratory Directed Research and Development Program of Lawrence Berkeley National Laboratory under the U.S. Department of Energy, Contract No. DE-ACO3-76SF00098, and used resources of the National Energy Research Scientific Computing Center, which is supported by the Office of Energy Research of the U.S. Department of Energy. A.R.L. was supported by the Royal Society. We thank Kevin Campbell and the NESRC Visualization Group, Graham Vincent at Sussex, and Konrad Kuijken and the Kapteyn Institute for help with data visualization.
[1] T. W. B. Kibble, J. Phys. A9, 1387 (1976).

[2] A. Vilenkin and E. P. S. Shellard, Cosmic Strings and Other Topological Defects, Cambridge University Press, Cambridge (1994); M. Hindmarsh and T. W. B. Kibble, Rep. Prog. Phys. 58, 477 (1995).

[3] B. Allen, R.R. Caldwell, S. Dodelson, L. Knox,E.P.S. Shellard, and A. Stebbins, Phys. Rev. Lett. 79, 2624 (1997); U.-L. Pen, U. Seljak and N. Turok, Phys. Rev. Lett. 79, 1611 (1997); A. Albrecht, R. A. Battye, J. Robinson, astro-ph/9711121

[4] W.H. Zurek, Nature 317, 505 (1985)

[5] See, for instance, Formation and Interactions of Topological Defects, NATO-ASI series B, Physics, vol. 349, eds. A.C. Davis and R. Brandenberger, Plenum Press 1995.

[6] C. Bäuerle, Yu.M. Bunkov, S.N. Fisher, H. Godfrin and G.R. Pickett, Nature 382, 332 (1996); V.M.H. Ruutu, V.B. Eltsov, A.J. Gill, T.W.B. Kibble, M. Krusius, Yu.G. Makhlin, B. Plaçais, G.E. Volovik and Wen Xu, Nature 382, 334 (1996).

[7] S. Rudaz and A.M. Srivastava, Mod. Phys. Lett A8, 1443 (1993); M. Hindmarsh, A.C. Davis and R. Brandenberger, Phys. Rev D49, 1944 (1994); T.W.B. Kibble and A. Vilenkin, Phys. Rev. D52, 679 (1995).

[8] E. Copeland and P.Saffin, Phys. Rev. D54, 6088 (1996); P.Saffin and E. Copeland, Phys. Rev. D56, 1215 (1997).

[9] Y. Nambu, Nucl. Phys. B130, 505 (1977); T. Vachaspati, Phys. Rev. Lett. 68, 1977 (1992), (E) 69, 216 (1992), Nucl. Phys. B397, 648 (1993).

[10] T. Vachaspati and A. Achúcarro, Phys. Rev D 44, 3067 (1991).

[11] M. Hindmarsh, Phys. Rev. Lett. 68, 1263 (1992).

[12] M.E. Zhitomirskii, JETP Lett. 49, 379 (1989); M. Palumbo, P. Muzikar and J.A. Sauls, Phys. Rev. B42, 2681 (1990); M.E. Zhitomirskii, JETP Lett. 55, 472 (1992); M. Palumbo and P. Muzikar, Phys. Rev. B45, 12620 (1992); A. Achúcarro and J.L. Mañes, Solid State Comm. 99, 831 (1996).

[13] A. Achúcarro, J. Borrill and A. R. Liddle, Phys. Rev. D57, 3742 (1998).

[14] A. Achúcarro, J. Borrill and A. R. Liddle, Report No. hep-ph/9802306, submitted to Phys. Rev. Lett.

[15] I. Burlachkov and N.B. Kopnin, Sov. Phys. JETP 65, 630 (1987)

[16] G.E. Volovik, Soviet Phys. Usp.27, 363 (1984)

[17] T. Vachaspati and A. Vilenkin, Phys. Rev. D30, 2036 (1984).

[18] A. Achúcarro, K. Kuijken, L. Perivolaropoulos and T. Vachaspati, Nucl. Phys. B388, 435 (1992).

[19] M. Hindmarsh, Nucl. Phys. B392, 461 (1993).

[20] M. Nagasawa and J. Yokoyama, Phys. Rev. Lett. 77, 2166 (1996).

[21] A. Achúcarro, J. Borrill and A. R. Liddle, in preparation.

[22] A.A. Abrikosov, Sov. Phys. JETP 5, 1174 (1957).

[23] H. B. Nielsen and P. Olesen, Nucl. Phys. B61, 45 (1973).

[24] G. W. Gibbons, M. E. Ortiz, F. Ruiz Ruiz and T. M. Samols, Nucl. Phys. B385, 127 (1992).

[25] K.. Benson and M. Bucher, Nucl. Phys. B406, 355 (1993).

[26] R. Leese and T Prokopec, Phys. Rev. D 44, 3749 (1991); T. Prokopec, Phys. Lett. B 262, 215 (1991).

[27] J. Ye and R. H. Brandenberger, Nucl. Phys. B346, 149 
(1990).

[28] R. H. Brandenberger and A. C. Davis, Phys. Lett. B308, 79 (1993).

[29] M. James, L. Perivolaropoulos and T. Vachaspati, Nucl. Phys. B395, 534 (1993); W. Perkins, Phys. Rev. D 47, R5224 (1993). 